\title{
Minimesohepatectomy for Colorectal Liver Metastasis Invading the Middle Hepatic Vein at the Hepatocaval Confluence
}

Guido Torzilli, MD, PhD, Florin Botea, MD, Matteo Donadon, MD, Matteo Cimino, MD, Daniele Del Fabbro, MD, and Angela Palmisano, MD

Liver Surgery Unit, Third Department of Surgery, University of Milan School of Medicine, IRCCS Istituto Clinico Humanitas, Rozzano, Milan, Italy

\begin{abstract}
Background. In case of liver tumors invading the middle hepatic vein (MHV) at the hepatocaval confluence (HC) major resection is recommended. We describe a new ultrasound-guided conservative operation for patients with colorectal liver metastasis (CLM) invading the MHV at the HC.

Methods. The case of a 65-year-old woman with two CLMs is described. One CLM was in segments 4-superior (S4-superior) and 8-ventral (S8-ventral) with invasion of the MHV $2 \mathrm{~cm}$ from the HC, while the other was in segment 8-dorsal (cranial portion). J-shaped laparotomy and intraoperative ultrasonography (IOUS) were carried out. Anterior surface of the $\mathrm{HC}$ was exposed, and compression using the surgeon's fingertips was applied at the MHV. Once reversal flow in the peripheral portion of the MHV, and/or shunting collaterals with right or left hepatic vein, and/or hepatopetal flow in portal branches to right
\end{abstract}

paramedian section (P5-8) and/or to segment 4-inferior (P4-inferior) were detected by color Doppler IOUS (CDIOUS), partial resection of S4-superior and S8-ventral with vascular resection of MHV was performed.

Results. The disclosure of those three criteria by CDIOUS enables the performance of minimesohepatectomy. No congestion of the remnant liver was found. Ninety-day mortality and morbidity were nil. The patient was discharged 8 days after surgery. At 11 months of follow-up the patient underwent percutaneous radiofrequency ablation for a new 15-mm CLM in segment-8-dorsal (caudal portion). Currently, the patient is alive and free of disease at 17 months after surgery.

Conclusions. The use of CD-IOUS may allow conservative resection of liver tumors invading the MHV at the HC. This might limit the need for larger resections, and broadens the role of IOUS in optimizing surgical strategy.

Electronic supplementary material The online version of this article (doi:10.1245/s10434-009-0728-6) contains supplementary material, which is available to authorized users.

(C) Society of Surgical Oncology 2009

First Received: 28 April 2009;

Published Online: 23 October 2009

G. Torzilli, $\mathrm{MD}, \mathrm{PhD}$

e-mail: guido.torzilli@unimi.it 